\title{
Conformation of a Diblock or Random Square-Well Copolymer in Dilute
}

\author{
Solution \\ Jun Cai and John M. Prausnitz ${ }^{\text {a) }}$ \\ Department of Chemical Engineering, University of California, Berkeley, and Chemical Sciences \\ Division, Lawrence Berkeley National Laboratory, Berkeley, California 94720
}

\begin{abstract}
Combined with an approximation suggested by Taylor and Lipson for the third-order correlation function and the translational invariance approximation, the BGY equation is used to calculate the conformation and thermodynamic properties of a diblock square-well copolymer or a random square-well copolymer. The BGY results for the end-to-end distribution of copolymers are in good agreement with Monte Carlo simulations when the attractive interaction between segments is not strong. BGY calculations semi-quantitatively predict the conformations of a copolymer when the attractive interaction is strong. At low temperatures, one block comprised of attractive segments of the diblock copolymer collapses while the other block, comprised of repulsive segments, dominates the scaling behavior. The end-to-end-distance distribution function for a random copolymer is similar to that for an $\mathrm{AB}$... AB copolymer when the temperature is at least moderate.
\end{abstract}

\section{INTRODUCTION}

Folding and unfolding (denaturing) of proteins is a well-known but incompletely understood phenomenon. The simplest model to describe the denaturation of proteins, the coil-globule

a) Author to whom correspondence should be addressed. Email: prausnit@,cchem.berkeley.edu. Phone: 1-510-642-3592. Fax: 1-510-642-4478 
transition of a polymer, has received much attention in the past two decades. ${ }^{1}$ A single homopolymer chain in dilute solution shows three types of configuration depending on the solvent: ${ }^{2-5}$ conformations of a single polymer chain are classified as expanded in a good solvent, ideal (Gaussian-chain-like) in a theta solvent, and compact in a poor solvent. When the solvent changes from good to poor, a coil-to-globule transition occurs if the molecular weight of the polymer is large. The globule state can be divided into two subclasses: the random globule and the frozen globule (at relatively lower temperature) mimic the folding of a protein. ${ }^{1}$ Although there are some subtle, unsolved problems (e.g. the logarithmic correction to the scaling law at the theta temperature ${ }^{6-8}$ ), the conformations of a homopolymer are relatively simple and well understood. $^{9-15}$ It is more difficult to describe the complex configuration of a copolymer, even for the simplest case where the copolymer contains only two kinds of segments. ${ }^{16,17}$ However, for understanding proteins, a copolymer provides a better simplified model than a homopolymer. In this work we present some theoretical results for the conformation of a block or random copolymer in dilute solution.

For a theoretical study of polymer collapse, the most widely used theory is the self-consistent field theory (SCF) of Edwards ${ }^{18,19}$ that leads to a diffusion or Schrödinger-like equation. In self-consistent field theory, the polymer is modeled as an elastic chain with interactions between segments. A hierarchy of Green functions is obtained. Approximations are needed to truncate the hierarchy. Using renormalization-group theory and diagram expansion, self-consistent field theory is able to describe the coil-globule transition of a hompolymer close to the theta point. ${ }^{9}$ With the help of a saddle-point approximation, self-consistent field theory can describe the adsorption of random copolymers on a solid surface. ${ }^{20}$ Because the self-consistent field theory is concerned with a very long polymer, it omits details of the interactions between segments and therefore can only describe the globule state close to the theta point. 
An alternative to SCF theory is to take advantage of the theory of spin glasses. ${ }^{21,22}$ With the help of the replica trick and replica symmetry breaking, ${ }^{23,24}$ we can find the frozen globule state of a random copolymer averaged over all possible sequences of the segments. ${ }^{25-28}$ Unlike the random globule, the state of the frozen globule is usually unique and corresponds to the global minimum of the free energy. Therefore, it can describe the folding state of a protein and encoding. ${ }^{28}$ For a fixed-sequence random copolymer, Allegra and Gonazzoli ${ }^{16,17,29,30}$ developed a free-energy model that shows both the random and frozen globule states for random copolymers with fixed sequences of segments.

For a more realistic segment-segment potential model, (e.g. a square-well chain or a Lennard-Jones chain), the integral-equation theory of liquids is best. ${ }^{10-13,31,32}$ An advantage of integral-equation theory is that its results can be compared with Monte Carlo (MC) simulations. Based on a new version of the Kirkwood superposition approximation, Eu and $\mathrm{Gan}^{12,13}$ derived a hierarchy of correlation functions for molecular liquids that predict the scaling law when the temperature is not very low. Another well-known integral equation for a single polymer chain is the classic Born-Green-Yvon (BGY) equation. ${ }^{33,34}$ After introducing some approximations to truncate the infinite hierarchy, Taylor and Lipson ${ }^{10}$ showed that the BGY equation provides good agreement with the scaling law for a homopolymer with repulsion interactions only. This theory gives a semi-quantitative description of the collapse of a square-well chain. Compared to Eu and Gan's theory, BGY provides a simpler mathematical expression. For a single chain in the bulk, because only a first-order differential equation is used, BGY avoids the difficulty of convergence for finding the roots of the non-linear equations in Eu and Gan's theory.

In this work, we use the BGY integral equation to investigate the coil-globule transition of a diblock copolymer and a random copolymer. Our results are a direct consequence of the work for homopolymers in Reference 10. In Section II, we introduce briefly the BGY integral-equation 
theory for a single copolymer and for a random copolymer. As shown by Taylor and Lipson, ${ }^{10}$ BGY cannot predict the frozen globule state of a square-well chain. Therefore, we focused on the coil-globule transition. In Section III, we introduce the model and an MC simulation method. In Section IV, we calculate conformation properties for a diblock copolymer and for a random copolymer comprised of two kinds of segments.

\section{THEORY}

\section{IIa. BGY Equation for a Copolymer with a Fixed Sequence}

We apply Taylor and Lipson's work ${ }^{10}$ for a homopolymer chain to a copolymer chain. The polymer is modeled as a freely jointed chain whose segments are hard spheres with short-range attraction between non-bonded segments. The segments are not necessarily the same. The solvent is a continuous medium; interactions between solvent and polymer are integrated into the effective interaction between segments. The canonical partition function of a single chain containing $N$ segments is given by:

$$
Z_{N}=V^{-1} \int \cdots \int \prod_{i=1}^{N-2} \prod_{j=i+2}^{N} \exp \left(-\beta u_{i j}\right) \prod_{i=1}^{N-1} s_{i, i+1} \prod_{i=1}^{N} \mathrm{~d} \mathbf{r}_{i}
$$

where $V$ is the volume of the system; subscripts $i, j$ are the ordinal number of the segments; $u_{i j}$ is the non-bonding interaction energy between segments $i$ and $j ; s_{i, i+1}$ is the Boltzmann factor for bonding energy between segments $i$ and $i+1$. The Boltzmann factor for bonding energy is given by:

$$
S_{i, i+1}\left(\mathbf{r}_{i}, \mathbf{r}_{i+1}\right)=\frac{1}{4 \pi \sigma_{i, i+1}^{2}} \delta\left(\left|\mathbf{r}_{i}-\mathbf{r}_{i+1}\right|-\sigma_{i, i+1}\right) ; \quad \sigma_{i j}=\frac{1}{2}\left(\sigma_{i}+\sigma_{j}\right)
$$

where $\delta$ is the Dirac function and $\sigma_{i}$ is the diameter of the hardcore of segment $i$. The factor 
$V^{-1}$ in Eq. (1) is added for consistency with the definition of the intra-molecular distribution function defined by: ${ }^{12}$

$$
w_{k l}\left(r_{k l}\right)=Z_{N}^{-1} \int \cdots \int \prod_{i=1}^{N-2} \prod_{j=i+2}^{N} \exp \left(-\beta u_{i j}\right) \prod_{i=1}^{N-1} s_{i, i+1} \prod_{i \neq k, l}^{N} \mathrm{~d} \mathbf{r}_{i} .
$$

where $r_{k l}$ is the distance between segments $k$ and $l$. Due to the connection between segments, $w_{k l}(r)$ vanishes when $r$ is larger than the entire length of all bonds between segments $k$ and $l$. The intra-molecular distribution function satisfies the normalization condition:

$$
\int \mathrm{d} r 4 \pi r^{2} w_{k l}(r)=1
$$

Calculating the gradient of the intra-molecular distribution function in Eq. (3) with respect to the position vector of the segment $l$, we obtain the BGY equation of a single chain:

$$
\nabla_{l} w_{k l}=-w_{k l} \nabla_{l} \beta u_{k l}-\sum_{j \neq k, l, l \pm 1} \int_{\mathrm{d}} \mathrm{d} \mathbf{r}_{j} w_{j k l} \nabla_{l} \beta u_{j l}+\int \mathrm{d} \mathbf{r}_{l-1} w_{k, l-1, l} \nabla_{l} \ln s_{l-1, l}+\int \mathrm{d} \mathbf{r}_{l+1} w_{k, l, l+1} \nabla_{l} \ln s_{l, l+1} .
$$

where $w_{j k l}$ is the third-order intra-molecular distribution function among segments $j, k$ and $l$. In this work, we focus on the end-to-end distribution function $w_{1 N}(r)$. For the end-to-end distribution function, Eq. (5) becomes:

$$
\nabla_{1} w_{1 N}=-w_{1 N} \nabla_{1} \beta u_{1 N}-\sum_{j=3}^{N-1} \int \mathrm{d} \mathbf{r}_{j} w_{1 j N} \nabla_{1} \beta u_{1 j}+\int \mathrm{d} \mathbf{r}_{2} w_{12 N} \nabla_{1} \ln s_{12}
$$

Eq. (6) is used in Taylor and Lipson's work. ${ }^{10}$ Eq. (6) concerns the gradient with respect to the position vector of segment 1. We also have another equation similar to Eq. (6) for the gradient with respect to the position vector of the last segment $N$. These two equations are equivalent because the BGY equation is rigorous. After the approximation Eq. (9) is introduced, the two versions of Eq. (6) are still equivalent for a homopolymer but not for a copolymer. To make the approximation described later consistent with the BGY equation, we introduce two variables to 
replace the position vectors of end segments and transform the original BGY equation Eq. (6). The two variables are:

$$
\left\{\begin{array} { l } 
{ \mathbf { r } _ { \mathrm { d } } = \mathbf { r } _ { 1 } - \mathbf { r } _ { N } } \\
{ \mathbf { r } _ { \mathrm { c } } = \mathbf { r } _ { 1 } + \mathbf { r } _ { N } }
\end{array} \text { or } \left\{\begin{array}{l}
\mathbf{r}_{1}=\left(\mathbf{r}_{\mathrm{c}}+\mathbf{r}_{\mathrm{d}}\right) / 2 \\
\mathbf{r}_{N}=\left(\mathbf{r}_{\mathrm{c}}-\mathbf{r}_{\mathrm{d}}\right) / 2
\end{array}\right.\right.
$$

We calculate the gradient of Eq. (3) with respect to $\mathbf{r}_{\mathrm{d}}$ rather than with respect to $\mathbf{r}_{1}$ or $\mathbf{r}_{N}$ and obtain:

$$
\begin{aligned}
\nabla_{\mathrm{d}} w_{1 N}= & w_{1 N} \nabla_{\mathrm{d}}\left(-\beta u_{1 N}\right)+\sum_{j=3}^{N-2} \int \mathrm{d} \mathbf{r}_{j} w_{1 j N} \nabla_{\mathrm{d}}\left(-\beta u_{1 j}-\beta u_{j N}\right) \\
& +\int \mathrm{d} \mathbf{r}_{N-1} w_{1, N-1, N} \nabla_{\mathrm{d}}\left(\ln s_{N-1, N}-\beta u_{1, N-1}\right)+\int \mathrm{d} \mathbf{r}_{2} w_{12 N} \nabla_{\mathrm{d}}\left(\ln s_{12}-\beta u_{2, N}\right)
\end{aligned}
$$

Eq. (8) is not useful unless we know the third-order distribution function. To calculate the third-order distribution function, we use the approximation suggested by Taylor and Lipson ${ }^{10}$ with a small change suitable for a copolymer:

$$
\begin{aligned}
& w_{1 i N}=w_{1 i} w_{i N} \Gamma_{1 i N}\left(r_{1 N}\right) \\
& \Gamma_{1 i N}\left(r_{1 N}\right)= \begin{cases}\frac{A_{N} w_{1 N}\left(r_{1 N}\right)}{\int \mathrm{d} \mathbf{r}_{i} w_{1 i} w_{i N}}, & r_{1 N} \leq \sigma_{1}+\sigma_{i}+\sigma_{N} . \\
\Gamma_{1 i N}\left(\sigma_{1}+\sigma_{i}+\sigma_{N}\right), & r_{1 N}>\sigma_{1}+\sigma_{i}+\sigma_{N}\end{cases}
\end{aligned}
$$

where $A_{N}$ is a constant to make the distribution function $\rho_{1 N}(r)(N>4)$ vanish when the chain is fully stretched i.e. at $r=\sigma_{1 N}+\sum_{i=2}^{N-1} \sigma_{i}$. It can be shown that Eq. (9) is exact for any trimer in the bulk, i.e.

$$
w_{123}=Z_{3}^{-1} s_{12} s_{23} \exp \left(-\beta u_{13}\right)=\frac{w_{12} w_{23} w_{31}}{\int \mathrm{d}_{2} w_{12} w_{23}}
$$

Substituting Eqs. (2) and (10) into Eq. (8), after some rearrangement, ${ }^{35}$ Eq. (8) becomes 


$$
\begin{aligned}
& \frac{\mathrm{d} w_{1 N}(r)}{\mathrm{d} r}=-w_{1 N}(r) \frac{\mathrm{d} \beta u_{1 N}(r)}{\mathrm{d} r}+\frac{\Gamma_{12 N}}{8 \sigma_{12}^{2} r^{2}}\left[\begin{array}{l}
-\frac{\mathrm{d}}{\mathrm{d} t}\left[\int_{|r-t|}^{r+t} \mathrm{~d} u \cdot u\left(r^{2}+t^{2}-u^{2}\right) w_{1, N-1}(u)\right]_{t=\sigma_{12}} \\
+\int_{0}^{+\infty} \mathrm{d} t \cdot w_{2 N}(t)\left[-\beta u_{2 N}^{\prime}(t)\right] \sigma_{12}\left(r^{2}+t^{2}-\sigma_{12}^{2}\right)
\end{array}\right] \\
& +\sum_{j=3}^{N-2} \frac{\pi \Gamma_{1 j N}(r)}{2 r^{2}}\left[\begin{array}{l}
\int_{0}^{+\infty} \mathrm{d} t \cdot w_{1 j}(t)\left[-\beta u_{1 j}^{\prime}(t)\right] \int_{|r-t|}^{r+t} \mathrm{~d} u \cdot u\left(r^{2}+t^{2}-u^{2}\right) w_{j N}(u) \\
+\int_{0}^{+\infty} \mathrm{d} t \cdot w_{j N}(t)\left[-\beta u_{j N}^{\prime}(t)\right] \int_{|r-t|}^{r+t} \mathrm{~d} u \cdot u\left(r^{2}+t^{2}-u^{2}\right) w_{1 j}(u)
\end{array}\right] \\
& +\frac{\Gamma_{1, N-1, N}}{8 \sigma_{N-1, N}^{2} r^{2}}\left[\begin{array}{l}
-\frac{\mathrm{d}}{\mathrm{d} t}\left[\int_{|r-t|}^{r+t} \mathrm{~d} u \cdot u\left(r^{2}+t^{2}-u^{2}\right) w_{1, N-1}(u)\right]_{t=\sigma_{N-1, N}} \\
+\int_{0}^{+\infty} \mathrm{d} t \cdot w_{1, N-1}(t)\left[-\beta u_{1, N-1}^{\prime}(t)\right] \sigma_{N-1, N}\left(r^{2}+t^{2}-\sigma_{N-1, N}^{2}\right)
\end{array}\right]
\end{aligned}
$$

Eq. (11) concerns the intra-molecular distribution function. Rigorously, the intra-molecular distribution function should be found by solving Eq. (5). However, for a chain of $N$ segments, there are $O\left(N^{2}\right)$ intra-molecular distribution functions. It is tedious and computer-time-consuming to solve these differential equations simultaneously. For simplicity, we use an assumption similar to translational invariance of the intra-molecular distribution function: ${ }^{13}$ we assume that the intra-molecular density distribution function of a $N$-segment chain $w_{k l}(r)$ is equal to the end-to-end distribution function of a $|k-l+1|$-segment chain. Since our target is a copolymer, the sequence of this $|k-l+1|$-segment chain should be the same as that of the corresponding part of the target chain. Eq. (11) is a first-order ordinary differential equation that can be integrated directly. The initial condition, i.e. the contact value of the distribution function, is determined by the normalization condition Eq. (4). Because Eq. (11) is linear, we can write the analytic solution. If the hardcore is the same for all segments, then there exists a solution: 


$$
\rho_{1 N}(r)=\left\{\begin{array}{l}
\rho_{1 N}(\sigma) \exp \left\{-\beta u_{1 N}(r)+\beta u_{1 N}(\sigma)+\int_{\sigma}^{r} \mathrm{~d} t f_{1}(t)\right\} \quad r \leq 3 \sigma \\
\rho_{1 N}(3 \sigma)+\int_{3 \sigma}^{r} \mathrm{~d} t f_{2}(t) ; \quad r>3 \sigma
\end{array}\right.
$$

where $f_{2}$ stands for the last three term in Eq. (11) and $f_{1}=f_{2} / \rho_{1 N}$. The end-to-end distribution of a trimer can be easily obtained from Eq. (3):

$$
w_{13}(r)=\frac{\exp \left[-\beta u_{13}(r)\right] \Theta\left(\sigma_{12}+\sigma_{23}-r\right)}{Z_{3} 8 \pi \sigma_{12} \sigma_{23} r}
$$

where $\Theta(r)$ is the Heaviside function. Eq. (13) can be used with Eq. (12) to calculate all end-to-end distribution functions of tetramers with various sequences of segments. Then, with the help of the end-to-end distribution functions of trimers and tetramers, we can obtain those for pentamers and so on.

Conformational and thermodynamic properties can be obtained from the intra-molecular distribution function. ${ }^{10}$ The average energy is given by:

$$
\langle\beta E\rangle=4 \pi \sum_{i=1}^{N-2} \sum_{j=i+2}^{N} \int_{0}^{+\infty} \mathrm{d} r \cdot r^{2} \beta u_{i j}(r) \rho_{i j}^{(2)}(r) .
$$

The average square end-to-end distance is given by

$$
\left\langle r_{1 N}^{2}\right\rangle=4 \pi \int_{0}^{+\infty} \mathrm{d} r \cdot r^{4} \rho_{1 N}^{(2)}(r)
$$

The radius of gyration is given by

$$
\left\langle R_{g}^{2}\right\rangle=\frac{1}{N^{2}} \sum_{i=1}^{N-1} \sum_{j=i+1}^{N}\left\langle r_{i j}^{2}\right\rangle
$$

\section{IIb. BGY Equation for a Random Copolymer}

For a random copolymer, the randomness comes from the disorder of the random sequence of different segments. The free energy and the partition function of a random copolymer are 
given by:

$$
-\beta F=\left\langle\ln Z_{N}\right\rangle_{\text {disorder }}
$$

where $F$ is the Helmholtz free energy; the brackets \langle\rangle$_{\text {disorder }}$ denote the average over all possible sequences; $Z_{N}$ is the partition function of the random copolymer with a fixed sequence. To calculate the end-to-end distribution function, we separate the non-bonding interaction into two parts: the repulsion (rep), which is the same for all segments, and the attraction (att), which depends on the species of interacting pairs, i.e.

$$
u_{i j}(r)=u_{i j}^{\mathrm{rep}}(r)+u_{i j}^{\mathrm{att}}(r)
$$

The end-to-end distribution function Eq. (3) can be expressed in another way, viz. by the functional derivative of the free energy with respect to the non-bonding repulsive interaction:

$$
w_{1 N}\left(\mathbf{r}_{1}, \mathbf{r}_{N}\right)=-\frac{\delta \beta F}{\delta \beta u_{1 N}^{\text {rep }}\left(\mathbf{r}_{1}, \mathbf{r}_{N}\right)}=\frac{\delta \ln Z_{N}}{\delta \beta u_{1 N}^{\text {rep }}\left(\mathbf{r}_{1}, \mathbf{r}_{N}\right)}
$$

Since the repulsions of all segment pairs are the same, the functional derivative in Eq. (19) is independent of the average \langle\rangle$_{\text {disorder }}$. Therefore, we can substitute Eq. (17) into Eq. (19) and obtain:

$$
\widetilde{w}_{1 N}\left(\mathbf{r}_{1}, \mathbf{r}_{N}\right)=\frac{\delta(-\beta F)}{\delta \beta u_{1 N}^{\text {rep }}\left(\mathbf{r}_{1}, \mathbf{r}_{N}\right)}=\left\langle w_{1 N}\left(\mathbf{r}_{1}, \mathbf{r}_{N}\right)\right\rangle_{\text {disorder }}
$$

where $\widetilde{w}_{1 N}$ is the end-to-end distribution function of the random copolymer averaged over the sequence disorder and $w_{1 N}$ is that of a fixed-sequence copolymer. The end-to-end distribution function $\widetilde{w}_{1 N}$ defined in Eq. (20) satisfied the BGY equation, Eq. (8). Eq. (20) shows that the end-to-end distribution function of a random copolymer is the average of the end-to-end distribution of all possible fixed-sequence copolymers. Similar statements hold for the properties given by Eqs. (14-16). For the Helmholtz free energy of a random copolymer, usually the replica 
trick is used. ${ }^{21}$ However, because the randomness we consider here comes from the random sequences, and because we obtain the energy $\langle\beta E\rangle_{\text {disorder }}$ from the correlation function of the random copolymer via Eq. (14), we need not use the replica trick. From the thermodynamic relation $\beta \partial F(\beta) / \partial \beta=E$, we obtain:

$$
\langle\beta F\rangle_{\text {disorder }}=\beta F^{0}+\int_{0}^{\beta}\langle E\rangle_{\text {disorder }} \mathrm{d} \beta
$$

where $\beta F^{0}$ is the free energy of the reference system at $\beta=0$, i.e. a hard chain. The free energy is useful to describe the random-globule-to-frozen-globule transition. However, approximations we used are only suitable for the disordered state of the polymer, we do not consider the coil globule-frozen globule transition in this work.

\section{MODEL AND SIMULATION}

We consider a square-well chain copolymer. The interaction energy $u_{i j}$ between non-bonded segments $i$ and $j$ is given by:

$$
\beta u_{i j}(r)=\left\{\begin{array}{c}
+\infty ; \quad r<\sigma_{i j} \\
\beta \varepsilon_{i j} ; \quad \sigma_{i j}<r<\lambda_{i j} \sigma_{i j} \\
0 ; \quad r>\lambda_{i j} \sigma_{i j}
\end{array}\right.
$$

where $\varepsilon_{i j}$ is the well depth and $\lambda_{i j}$ is a constant between 1 and 2 . The end-to-end distribution for a trimer square-well chain and the canonical partition function can be obtained directly from the definitions:

$$
w_{13}(r)=\frac{\left\{\Theta\left(r-\lambda_{13} \sigma_{13}\right)\left[1-\exp \left(-\beta \varepsilon_{13}\right)\right]+\exp \left(-\beta \varepsilon_{13}\right) \Theta\left(r-\sigma_{13}\right)\right\} \Theta\left(\sigma_{12}+\sigma_{23}-r\right)}{Z_{3} 8 \pi \sigma_{12} \sigma_{23} r}
$$




$$
Z_{3}=\left\{\begin{array}{c}
\frac{\left(\lambda_{13}^{2}-1\right) \sigma_{13}^{2} \exp \left(-\beta \varepsilon_{13}\right)+\left(\sigma_{12}+\sigma_{23}\right)^{2}-\lambda_{13}^{2} \sigma_{13}^{2}}{4 \sigma_{12} \sigma_{23}} ; \quad \sigma_{12}+\sigma_{23}>\lambda_{13} \sigma_{23} \\
\frac{\exp \left(-\beta \varepsilon_{13}\right)\left[\left(\sigma_{12}+\sigma_{23}\right)^{2}-\sigma_{13}^{2}\right]}{4 \sigma_{12} \sigma_{23}} ; \quad \sigma_{12}+\sigma_{23} \leq \lambda_{13} \sigma_{13}
\end{array}\right.
$$

The derivative of the potential with respect to $r$ is given by:

$$
\left[-\beta u_{i j}^{\prime}(r)\right]=\exp \left[-\beta \varepsilon_{i j}+\beta u(r)\right] \delta\left(r-\sigma_{i j}\right)+\beta \varepsilon_{i j} \delta\left(r-\lambda_{i j} \sigma_{i j}\right)
$$

When Eq. (11) is integrated, we need to know the correlation function $w_{i j}\left(\lambda_{i j} \sigma_{i j}\right)$ and how to calculate $w_{i j}(r) \beta u_{i j}^{\prime}(r)$. The derivation of Eq. (24), $w_{i j}\left(\lambda_{i j} \sigma_{i j}\right)$ and $w_{i j}(r) \beta u_{i j}^{\prime}(r)$ are given in the Appendix.

We use the Dickman-Hall algorithm ${ }^{36}$ for the canonical Monte-Carlo simulations. The procedure for simulation is as follows. First, the initial configuration of the copolymer is generated by an off-lattice self-avoiding random walk. Second, the chain is subjected to a "translate-jiggle" movement. A bond is chosen randomly on the chain. Then, randomly choose either one end (e.g. segment $i$ ) of the bond to be stationary and make the other end of the bond (segment $i-1$ ) translate a certain distance. Then, normalize the distance between these two segments to be the bond length, i.e. normalize $r_{i-1, i}$ to $\sigma_{i-1, i}$. The sub-chain (segments $1,2 \ldots$ $i-2$ ) that is connected to segment $i-1$ is subjected to the same translation as that for segment $i-1$. The Metropolis algorithm is used to decide if the movement is accepted or not. Then, a procedure similar to moving segment $i-1$ is applied repeatedly to segment $i-2$ and so on until the end of the chain.

\section{RESULTS AND DISCUSSIONS}

In all calculations, the copolymers are comprised of two kinds of segments. The diameters of hard cores of both segments are the same, i.e. $\sigma_{\mathrm{A}}=\sigma_{\mathrm{B}}=\sigma$. The well-width parameter is 
assumed to be constant, $\lambda_{i j}=1.5, i, j=\mathrm{A}, \mathrm{B}$. The number of segment $i, i=\mathrm{A}, \mathrm{B}$ in one molecule is denoted by $n_{i}$. In all calculations, segment $\mathrm{A}$ is a hard sphere and the interaction between $\mathrm{A}$ and $\mathrm{B}$ is repulsive only, i.e. $\varepsilon_{\mathrm{AA}}=\varepsilon_{\mathrm{AB}}=0$. Because of limited memory and computer time, the maximum chain length we calculated is $N=192$. Taylor and Lipson ${ }^{10}$ have shown that the BGY equation combined with the approximations cannot predict the correct conformation of a homopolymer if the attractive interaction between segments is very strong. Therefore, we consider only cases where $\beta \varepsilon_{i j}>-10$. We investigate how the conformation of a single polymer depends on the ratio $n_{A} / n_{B}$. The two limiting cases, $n_{A} / n_{B}=0$ and $+\infty$, correspond, respectively, to two homopolymers, i.e. the hardchain (pure A) and the square-well chain (pure B). The results for homopolymers are calculated in Reference 10. Here we duplicated plots for homopolymers in Reference 10 to verify the correctness of our computer program and to show how the ratio $n_{A} / n_{B}$ influences the conformation of a copolymer. We found that the consistency between the simulation and the theory declines gradually with decreasing $n_{A} / n_{B}$. The two limiting cases $n_{A} / n_{B}=0$ and $+\infty$ can be used as a criterion. Since the two limiting cases have been investigated thoroughly in Reference 10 for the theory and in Reference 15 for Monte Carlo simulation, we did not perform a comparison between the theory and the Monte Carlo simulation over the whole space of the parameters. Instead, we just compare several cases as examples. For random copolymers, we assume that the randomness arises from the random sequences. We assume that the probabilities of all possible sequences are the same. We calculate the conformational properties for all possible sequences and then obtain the average according to Eq. (20).

Figs. 1a and $1 \mathrm{~b}$ show the end-to-end distribution function for five diblock 64-mers. In Fig. 1a 
the energy parameter is $\beta \varepsilon_{\mathrm{BB}}=-0.5$. As expected, the end-to-end distribution function gradually expands with increasing fraction of segment A. Comparing to the MC data, for a symmetric diblock copolymer or a hardsphere chain, the BGY results are consistent with Monte-Carlo simulation. When the attractive interaction is strong, BGY overestimates the collapse of the polymer. The larger the attractive interaction, the larger the deviation, as expected, from the approximation Eq. (9). When the polymer collapses, the volumetric repulsion plays a more important role and the approximation Eq. (9) is not suitable.

Fig. 2 shows how the radius of gyration for a 32-mer diblock copolymer depends on the reduced temperature $T^{*}$ defined by the reciprocal of reduced attraction energy between segments $\mathrm{B}$, i.e $T^{*}=-1 / \beta \varepsilon_{\mathrm{BB}}$. In this case, because only one block of the diblock copolymer is comprised of attractive segments, the collapse of the chain applies only to this attractive block. When the radius of gyration changes rapidly, the temperature corresponds to the collapse temperature. As expected, if the fraction of segment B increases, the corresponding collapse temperature rises.

Fig. 3 shows how the radii of gyration of various diblock copolymers depend on the chain length with energy parameter $\beta \varepsilon_{\mathrm{BB}}=-1$. Fig. 4 shows how the scaling parameter $2 v$ (in the scaling law $R_{g}^{2} \sim N^{2 v}$ ) depends on the fraction of segment A. Since the chain length (up to 192) in the calculation is not long enough, the scaling parameter in Fig. 4 provides only an approximation. Here a subtle problem arises. As we can expect the scaling law certainly depends on the sequence of the segment. If segment $\mathrm{A}$ and segment $\mathrm{B}$ distribute uniformly on the polymer, e.g. $\mathrm{AABAAB} . . \mathrm{AAB}$, the scaling parameter $2 v$ will fall between the two limiting cases i.e. a hard chain and a square-well chain. However for a diblock copolymer, we think that the attractive segments (B) on one block tend to aggregate together and repulsive segments (A) on the other 
block dominate the scaling behavior. Thus the scaling parameter should be the same as that of the homopolymers with weak attraction $\left(N^{2 v_{\mathrm{A}}}>>N^{2 v_{\mathrm{B}}}, v_{\mathrm{A}}>v_{\mathrm{B}}\right)$ when the chain length is very long. However in this work, the chain length is not long enough to verify these intuitive explanations. For a diblock copolymer, at the chain length of 192, the scaling behavior is still not obvious, while for a homopolymer the scaling behavior appears at the chain length around $40 .{ }^{10}$

Fig. 5 shows the end-to-end distribution function of short random copolymers. The ratio of two different segments is $n_{\mathrm{A}}: n_{\mathrm{B}}=1$. Fig. 5 is for a random 4-mer. The energy parameter is $\beta \varepsilon_{\mathrm{BB}}=-8$. Since $\beta \varepsilon_{\mathrm{AB}}=0$, sequences $\mathrm{ABBA}$ and $\mathrm{AABB}$ actually are homopolymers. We didn't use the exact results ${ }^{10,11}$ but Monte Carlo simulation to compare with the theory. The approximation suggested by Taylor and Lipson and the translational invariance approximation work equally well for a 4-mer copolymer as it did for a 4 -mer homopolymer. ${ }^{10}$ For any sequence of a 4-mer, BGY combined with Taylor and Lipson's approximation ${ }^{10}$ is in good agreement with Monte Carlo simulations even if the attractive interaction is very strong.

Fig. 6a shows the end-to-end distribution function of a random half A-half B 8-mer. The energy parameter is $\beta \varepsilon_{\mathrm{BB}}=-1$. Such a random copolymer has 38 possible sequences. Monte Carlo simulations are performed for each sequence and then Eq. (20) is used for average. Although the BGY result for a random 8-mer is in good agreement with MC data, it is not so good for some fixed sequence copolymer as shown in plot 1 in Fig. 6a. Fig. $6 \mathrm{~b}$ shows the end-to-end distribution function of a random copolymer with a fixed sequence generated randomly by computer. The sequence is: AAABBABBAABAABAAABBBBBAAABBABBAB. The energy parameters are $\beta \varepsilon_{\mathrm{BB}}=-0.2$ or -1 . The results are similar to those for diblock copolymers. When the attractive interaction between two segments B increases, the polymer shrinks. When the attraction is not very strong, BGY-equation results are consistent with $\mathrm{MC}$ 
simulations. As shown by Taylor and Lipson, ${ }^{10} \mathrm{BGY}$ equation is in good agreement with $\mathrm{MC}$ results for short square-well homopolymer. However, for long chains, when the attractive energy is strong, due to the approximation Eq. (9) and the translational invariance approximation, the deviation is large. This problem is more serious for a random copolymer. Figs. $6 \mathrm{a}$ and $6 \mathrm{~b}$ shows that the theory qualitatively reflects the effect of sequences when the attraction is not weak.

Fig. 7 shows how the end-to-end distribution function of random copolymers depends on the attractive energy. The chain length is 16 and the ratio of two different segments is $n_{\mathrm{A}}: n_{\mathrm{B}}=1$. The energy parameters are $\beta \varepsilon_{\mathrm{BB}}=-1$ or -2 . The dashed line shows the end-end distribution function of an $\mathrm{AB}$... $\mathrm{AB}$ copolymer. The squares show the end-end distribution of an $\mathrm{AB}$ diblock copolymer. The chain shrinks when the attractive energy increases. The end-to-end distribution function of random copolymers is similar to that of an $\mathrm{AB}$... AB fixed-sequence copolymer if the attractive energy is moderate. This implies that when the polymer is not in a very compact collapse state, the thermodynamic properties of the random copolymer are similar to those of a copolymer with the fixed periodic sequence and the same ratio of two different segments. When the attractive interactions are strong, e.g. when $\beta \varepsilon_{\mathrm{BB}}=-2$, due to the collapse of the polymer, the conformation of the random copolymer is different from but still to some degree similar to that of an $\mathrm{AB}$... $\mathrm{AB}$ copolymer. However the conformation of a diblock copolymer is different from those of a random or $\mathrm{AB} \ldots \mathrm{AB}$ copolymer. For random copolymers or $\mathrm{AB} \ldots \mathrm{AB}$ copolymers, configurations change largely with changing attractive interactions, while for a diblock copolymer, such change is not so obvious. A diblock copolymer does not shrink as much as a random copolymer or $\mathrm{AB} . . \mathrm{AB}$ copolymer. This verifies to some degree our intuitive explanation for Figs. 3 and 4.

Fig. 8 shows how the radius of gyration for a random 12-mer depends on the reduced 
temperature $T^{*}$. The ratio of two different segments is $n_{\mathrm{A}}: n_{\mathrm{B}}=1$. The interactions between two segments $\mathrm{A}$ and between segments $\mathrm{A}$ and $\mathrm{B}$ are only repulsive, i.e. $\varepsilon_{\mathrm{AA}}=\varepsilon_{\mathrm{AB}}=0$. When the radius of gyration changes rapidly, the temperature corresponds to the collapse temperature. Similar to Fig. 7, the conformation of a random copolymer is similar to that of an $\mathrm{AB} \ldots \mathrm{AB}$ copolymer but not to a diblock copolymer. The collapse of the copolymer with uniformly distributed segments is stronger than those with other sequences.

\section{CONCLUSION}

To calculate the conformation properties and the thermodynamic properties for a diblock square-well copolymer or for a random square-well copolymer, we use the BGY equation combined with an approximation suggested by Taylor and Lipson for the third-order correlation function ${ }^{10}$ and the translational invariance approximation. ${ }^{13}$ The BGY results for the end-to-end distribution of a copolymer are in good agreement with Monte Carlo simulations when the attractive interaction between segments is not strong. BGY also semi-quantitatively predicts the conformations of a copolymer when the attractive interaction is strong. Due to the approximations, BGY overestimates the collapse when the attractive interaction is strong. Sequence effects on the conformation of a copolymer are qualitatively taken into account in the theory. Sequence effects are shown clearly in the comparison between the diblock copolymer, the $\mathrm{ABAB} \ldots \mathrm{AB}$ copolymer and the random copolymer. At low temperatures, when one block is comprised of attractive segments and the other block is only repulsions, the attractive block may collapse while the other block dominates the scaling behavior. However the end-to-end distance distribution function of random copolymers is similar to that of an $\mathrm{AB}$... AB copolymer when the temperature is at least moderate. Thus the scaling behavior may be between two limiting cases, 
i.e. a hardchain and a square-well chain. However, because the chain length is not long enough, the existence of the scaling law for a random copolymer is still not clear. Since the approximations we used are only suitable for a liquid-like polymer, the BGY equation cannot describe the frozen state of the polymer.

\section{Acknowledgement}

For financial support, the authors are grateful to the Office for Basic Sciences of the US Department of Energy and to the Chinese National Science Foundation.

\section{Appendix}

Derivation of Eq. (24), $w(r)\left[-\beta u^{\prime}(r)\right]$ and $w(\lambda \sigma)$

For simplicity, we omit subscripts. This omission will not give rise to ambiguity. The Boltzmann factor of the square-well potential, Eq. (21), is given by:

$$
\begin{aligned}
\exp [-\beta u(r)] & =\left\{\begin{array}{cc}
0 ; & r<\sigma \\
\exp (-\beta \varepsilon) ; & \sigma<r<\lambda \sigma \\
1 ; & r>\lambda \sigma
\end{array}\right. \\
& =\exp (-\beta \varepsilon) \Theta(r-\sigma)+[1-\exp (-\beta \varepsilon)] \Theta(r-\lambda \sigma) .
\end{aligned}
$$

The derivative of the Boltzmann factor is given by:

$$
\exp [-\beta u(r)] \cdot\left[-\beta u^{\prime}(r)\right]=\exp (-\beta \varepsilon) \delta(r-\sigma)+[1-\exp (-\beta \varepsilon)] \delta(r-\lambda \sigma) .
$$

Multiplying Eq. (A2) by $\exp [\beta u(r)]$ and integrating from $\lambda \sigma^{-}$to $\lambda \sigma^{+}$, we obtain

$$
\exp [\beta u(\lambda \sigma)]=\frac{\beta \varepsilon}{1-\exp (-\beta \varepsilon)} .
$$

Using the relationship $f(r) \delta(r-a)=f(a) \delta(r-a)$ and Eq. (A2), we obtain Eq. (24).

Because of the hardcore of the segment, the end-to-end distribution function can be written 
as:

$w(r)=w(r) \Theta(r-\sigma)$.

For $r<\lambda \sigma$, according to Eq. (A1), we have:

$w(r)=w(r) \exp [\beta \varepsilon-\beta u(r)]$

With the help of Eq. (24) and Eq. (A5), we have

$$
w(r)\left[-\beta u^{\prime}(r)\right]=w(r) \delta(r-\sigma)=w(\sigma) \delta(r-\sigma) ; r<\lambda \sigma
$$

Integrating Eq. (11) from $\lambda \sigma^{-}$to $\lambda \sigma^{+}$, we obtain two equations:

$$
\int_{\lambda \sigma-}^{\lambda \sigma+} \frac{w^{\prime}(r)}{w(r)} \mathrm{d} r=\int_{\lambda \sigma-}^{\lambda \sigma+}-\beta u^{\prime}(r) \mathrm{d} r \Rightarrow \frac{w\left(\lambda \sigma^{+}\right)}{w\left(\lambda \sigma^{-}\right)}=\exp (\beta \varepsilon)
$$

and

$$
\int_{\lambda \sigma-}^{\lambda \sigma+} w^{\prime}(r) \mathrm{d} r=\int_{\lambda \sigma_{-}}^{\lambda \sigma+}-\beta u^{\prime}(r) w(r) \mathrm{d} r \Rightarrow w\left(\lambda \sigma^{+}\right)-w\left(\lambda \sigma^{-}\right)=\beta \varepsilon \cdot w(\lambda \sigma) .
$$

Eqs. (A6-A8) are useful for integrating Eq. (11).

\section{REFERENCE CITED}

1. V. S. Pande, A. Yu Grosberg and T. Tanaka, Macromol. 28, 2218 (1995), J. Chem. Phys. 107, 5118 (1997), Folding \& Design, 2, 109 (1997).

2. A. Yu. Grosberg and A. R. Khokhlov, Statistical Physics of Macromolecules, American Institute of Physics, New York, 1994.

3. P. G. de Gennes, Scaling Concepts in Polymer Physics, Cornell University Press, Ithaca and London, 1979.

4. I. M. Lifshitz, A. Yu Grosberg and A. R. Khokhlov, Rev. Mod. Phys. 50, 683 (1978).

5. K. Freed, Renormalization Group Theory of Macromolecules, John Wiley and Sons, New 
York, 1987.

6. L. Duplantier, J. Chem. Phys. 86, 4233 (1987).

7. P. Grassberger and R. Hegger, J. Chem. Phys. 102, 6881 (1995).

8. J. Hager and L. Shafer, Phys. Rev. 60, 2071 (1999).

9. A. Kholodenko, K. Freed, J. Phys. A: Math. Gen. 17, 2703 (1984).

10. M. P. Taylor and J. E. G. Lipson, J. Chem. Phys. 104, 4835 (1996).

11. M. P. Taylor, Mol. Phys. 86, 73 (1995).

12. B. C. Eu and H. H. Gan, J. Chem. Phys. 99, 4804 (1993).

13. H. H. Gan and B. C. Eu, J. Chem. Phys. 99, 4103 (1993), 110, 3235 (1999).

14. Y. Zhou, C. Hall and M. Karplus, Phys. Rev. Lett. 77, 2822 (1996).

15. Y. Zhou, M. Karplus, J. Wichert and C. Hall, J. Chem. Phys. 107, 10691 (1997).

16. G. Allegra and F. Ganazzoli, J. Chem. Phys. 83, 397 (1985).

17. F. Ganazzoli, J. Chem. Phys. 108, 9924 (1998).

18. S. Edwards, Proc. Phys. Soc. (London) 85, 613 (1965).

19. K. Freed, Adv. Chem. Phys. 22, 1 (1972).

20. L. Gutman and A. Chakraborty, J. Chem. Phys. 101, 10074 (1994), 103, 10733 (1995).

21. S. Edwards and P. Anderson, J. Phys. F5, 965 (1975).

22. B. Derrida, Phys. Rev. Lett. 45, 79 (1980).

23. G. Parisi, J. Phys. A13, 1101 (1980), Phys. Rev. Lett. 50, 1946 (1983).

24. M. Merced and G. Parisi, J. Phys. (Paris) I1, 809 (1990).

25. E. Shakhnovich and A. Gutin, Biophys. Chem. 34, 187 (1989).

26. C. Sfatos, A. Gutin and E. Shakhnovich, Phys. Rev. E48, 465 (1993).

27. A. Chakraborty and E. Shakhnovich, J. Chem. Phys. 103, 10751 (1995).

28. E. Shakhnovich and A. Gutin, Nature 346, 773 (1990). 
29. G. Allegra and F. Ganazzoli, Adv. Chem. Phys. 75, 265 (1989).

30. G. Raos, G. Allegra and F. Ganazzoli, J. Chem. Phys. 100, 7804 (1994).

31. T. Hill, Statistical Mechanics, Dover, New York, 1956.

32. J. Hansen and I. McDonald, Theory of Simple Liquids, $2^{\text {nd }}$ ed., Academic Press, 1987.

33. M. Born and H. Green, Proc. R. Soc. London, Ser. A 188, 10 (1946).

34. S. Whittington and L. Dunfield, J. Phys. A6, 484 (1973).

35. P. Attard, J. Chem. Phys. 102, 5411 (1995).

36. R. Dickman and C. Hall, J. Chem. Phys. 85, 4108 (1986). 


\section{Captions}

Fig. 1a End-to-end distribution function of 64-mers with $\beta \varepsilon_{\mathrm{BB}}=-0.5$. Dots: $\mathrm{MC}$ data,

$n_{\mathrm{A}}+n_{\mathrm{B}}=64.1 . n_{\mathrm{A}} / n_{\mathrm{B}}=+\infty ; 2 . n_{\mathrm{A}} / n_{\mathrm{B}}=3 ; 3 . n_{\mathrm{A}} / n_{\mathrm{B}}=1 ; 4 . n_{\mathrm{A}} / n_{\mathrm{B}}=1 / 3 ; 5 . n_{\mathrm{A}} / n_{\mathrm{B}}=0$.

Fig. $1 \mathrm{~b}$ End-to-end distribution function of 64-mers. $\beta \varepsilon_{\mathrm{BB}}=-1$. Notation as in Fig. 1a

Fig. 2 Dependence of the radius of gyration of 32-mer on the reduced temperature.

$$
T^{*}=-1 / \beta \varepsilon_{\mathrm{BB}} . \text { Notation as in Fig. 1a }
$$

Fig. 3. Radius of gyration as a function of chain length with $\beta \varepsilon_{\mathrm{BB}}=-1$. Notation as in Fig.1a.

Fig. 4 The scaling parameter depends on the fraction of segment B. Chain length is up to 192.

$$
\beta \varepsilon_{\mathrm{BB}}=-1
$$

Fig. 5 End-to-end distribution function of 4-mers. $\beta \varepsilon_{\mathrm{BB}}=-8 . n_{\mathrm{A}} / n_{\mathrm{B}}=1$.

1. Random; 2. ABBA or AABB; 3. ABAB; 4. ABBA. Dots: Monte Carlo. Lines: Theory

Fig. 6a End-to-end density distribution of a random 8-mer with $\beta \varepsilon_{\mathrm{BB}}=-1$.

1. Fixed sequence 8-mer, BBAAAABB; 2. Random 8-mer. Line: theory. Dots: MC data.

Fig. 6b End-to-end distribution function of a fixed sequence random copolymer. Dots: MC data. 
1. $\beta \varepsilon_{\mathrm{BB}}=-1 ; 2 . \quad \beta \varepsilon_{\mathrm{BB}}=-0.2$.

Fig. 7 End-to-end distribution function of random copolymers.

1. $\beta \varepsilon_{\mathrm{BB}}=-2 ; 2 . \quad \beta \varepsilon_{\mathrm{BB}}=-1$. Solid lines: $\mathrm{BGY}$ for random copolymer. Dashed lines: BGY for AB...AB copolymer. Dots: $\mathrm{MC}$ for $\mathrm{AB}$... AB copolymer. Squares: $\mathrm{BGY}$ for diblock copolymer.

Fig. 8 Dependence of the radius of gyration on the reduced temperature. $T^{*}=-1 / \beta \varepsilon_{\mathrm{BB}}$.

1. Random; 2. AB...AB copolymer; 3. Diblock copolymer. Line: BGY for random copolymer.

Dots: $\mathrm{BGY}$ for an $\mathrm{AB} \ldots \mathrm{AB}$ copolymer. 
Fig. 1a J. Cai, J. Chem. Phys.

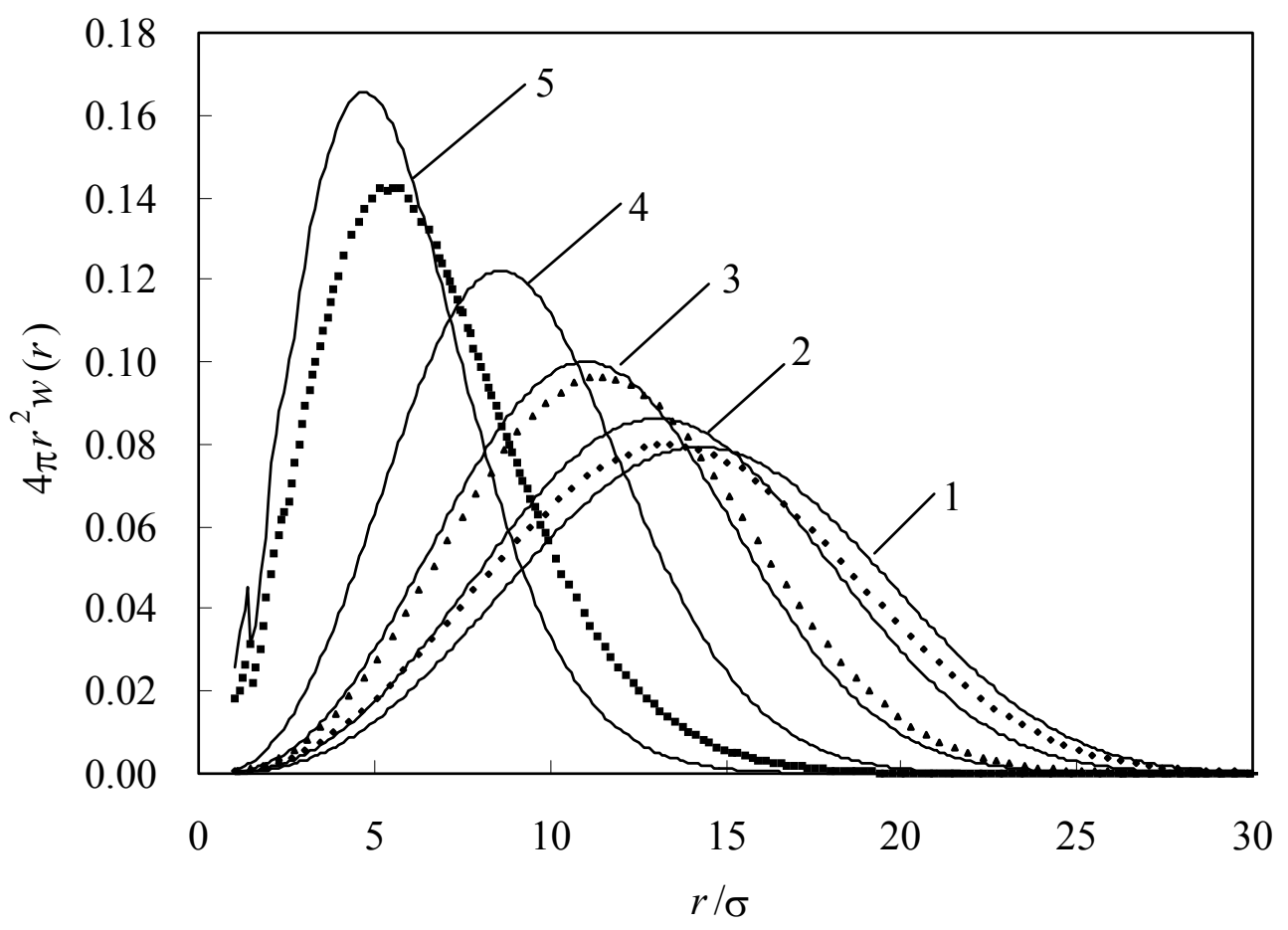


Fig. 1b J. Cai, J. Phys. Chem.

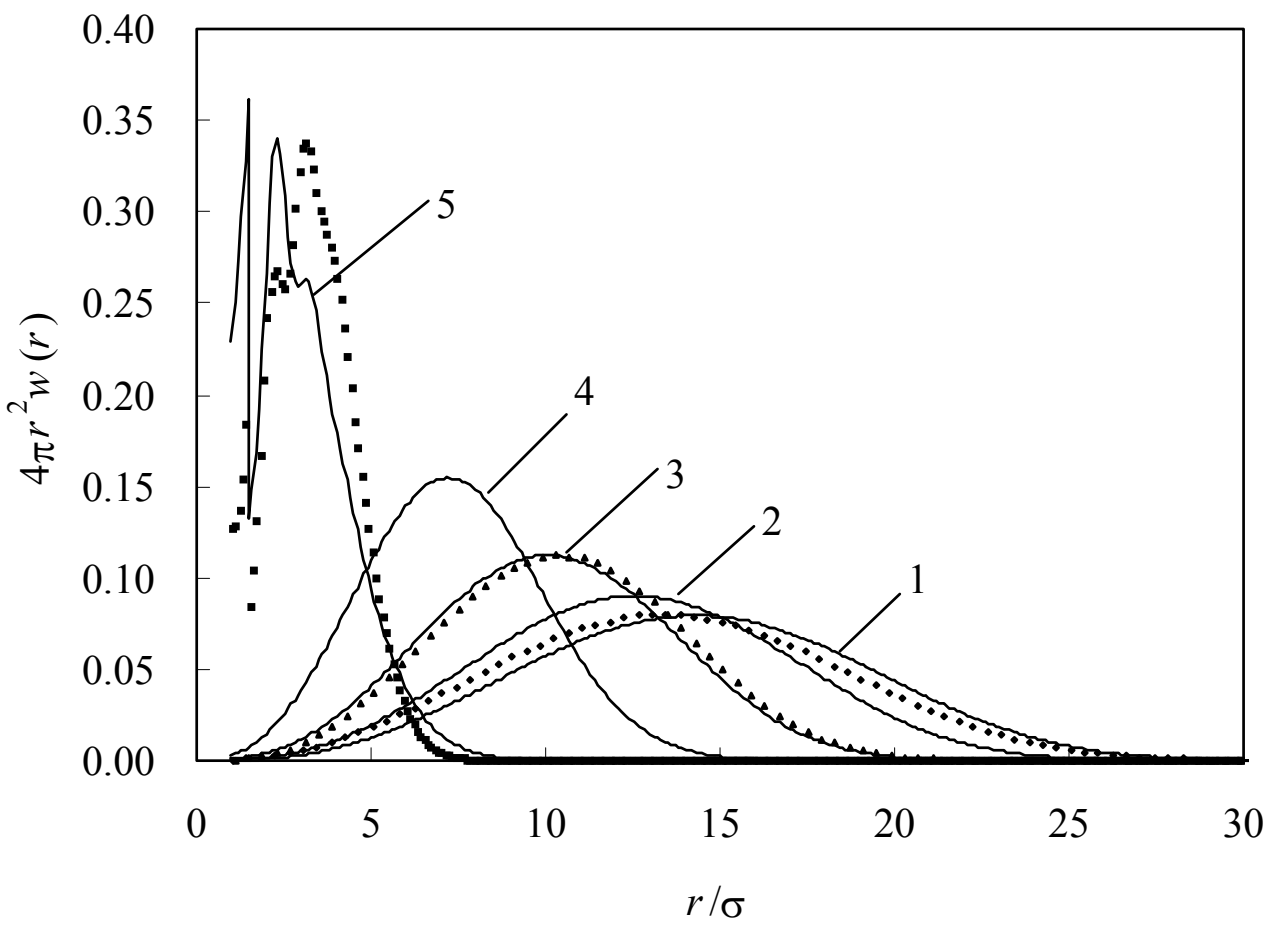


Fig. 2 J. Cai, J. Phys. Chem.

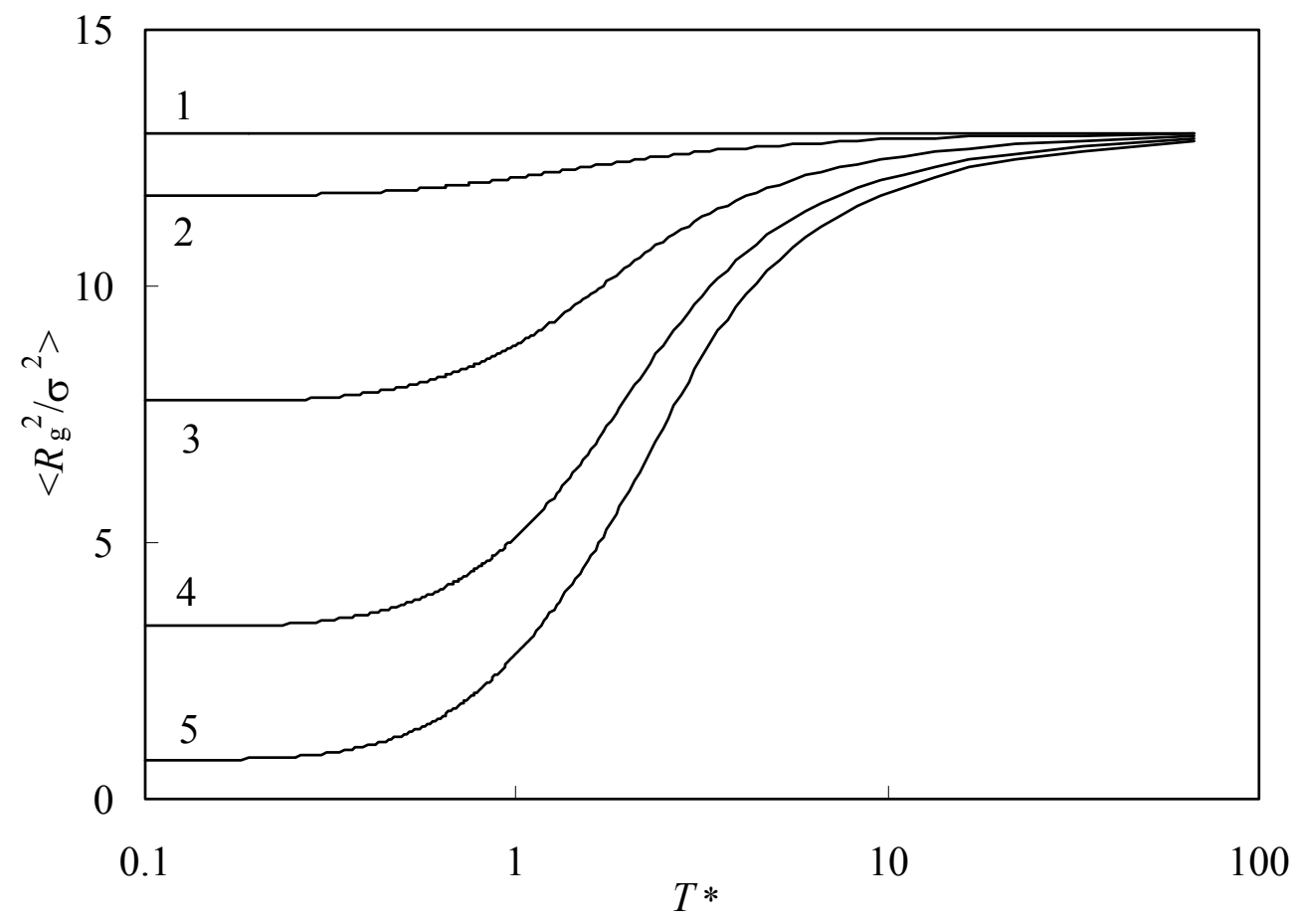


Fig. 3 J. Cai, J. Chem. Phys.

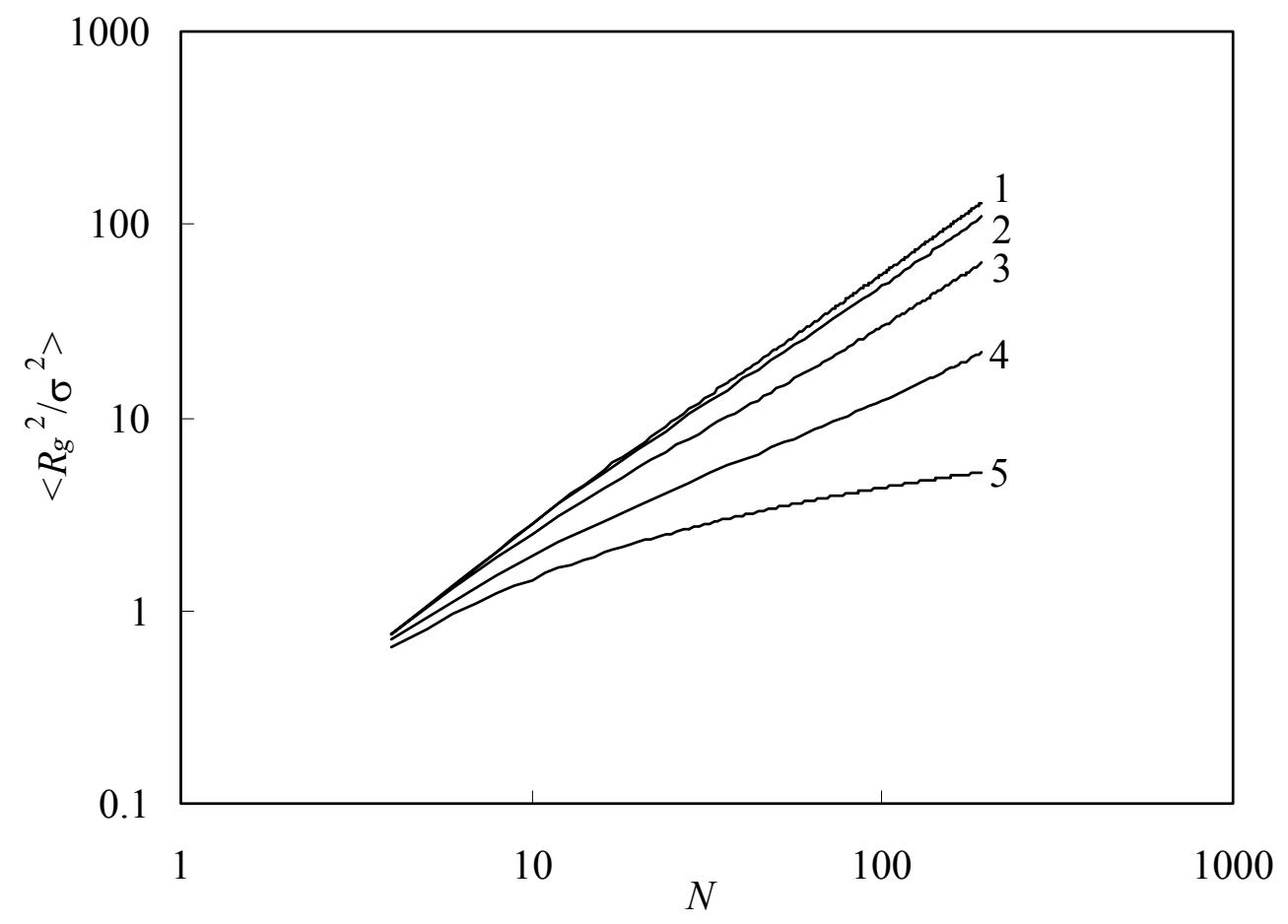


Fig. 4 J. Cai, J. Chem. Phys.

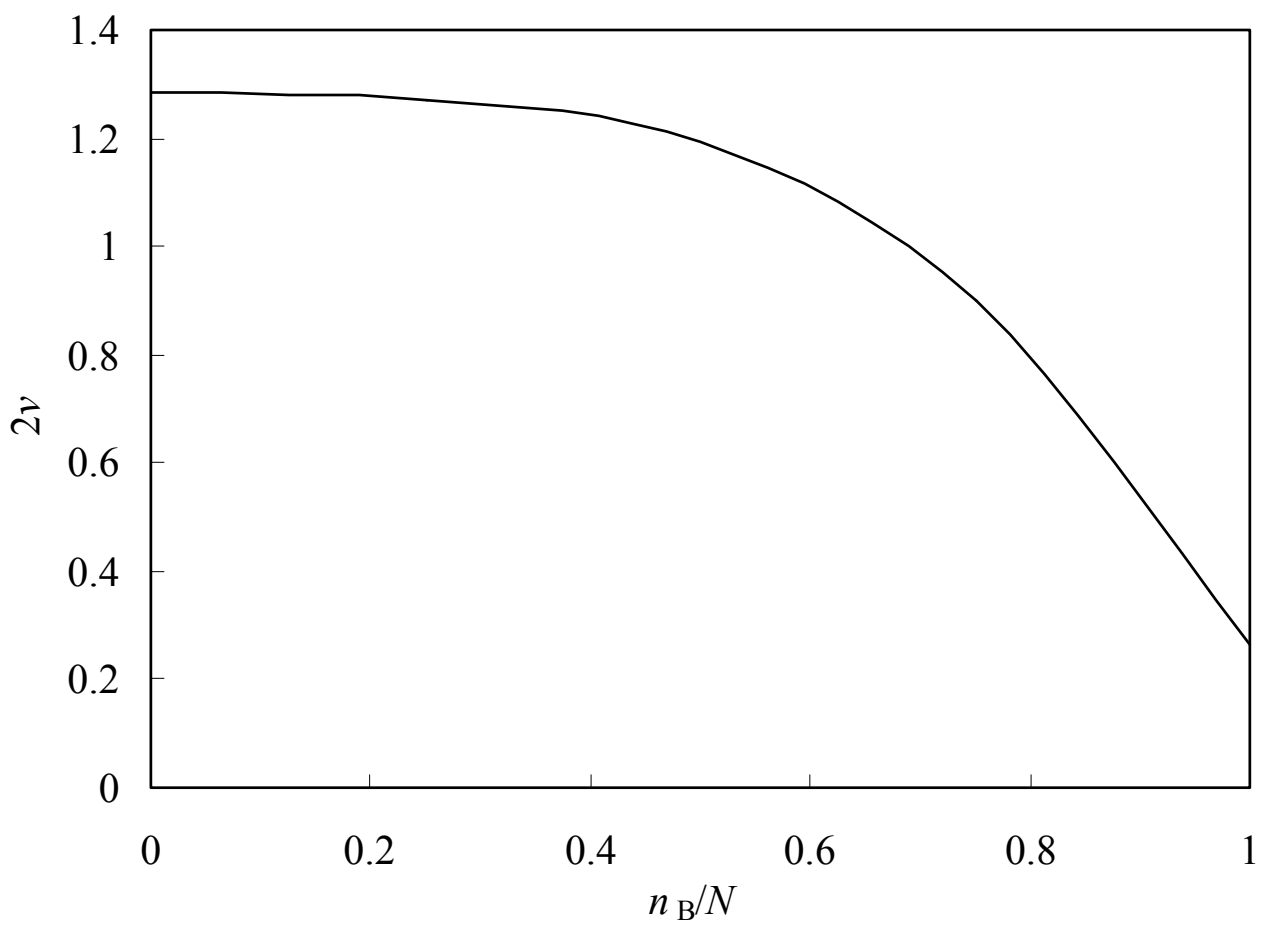


Fig. 5 J. Cai, J. Chem. Phys.

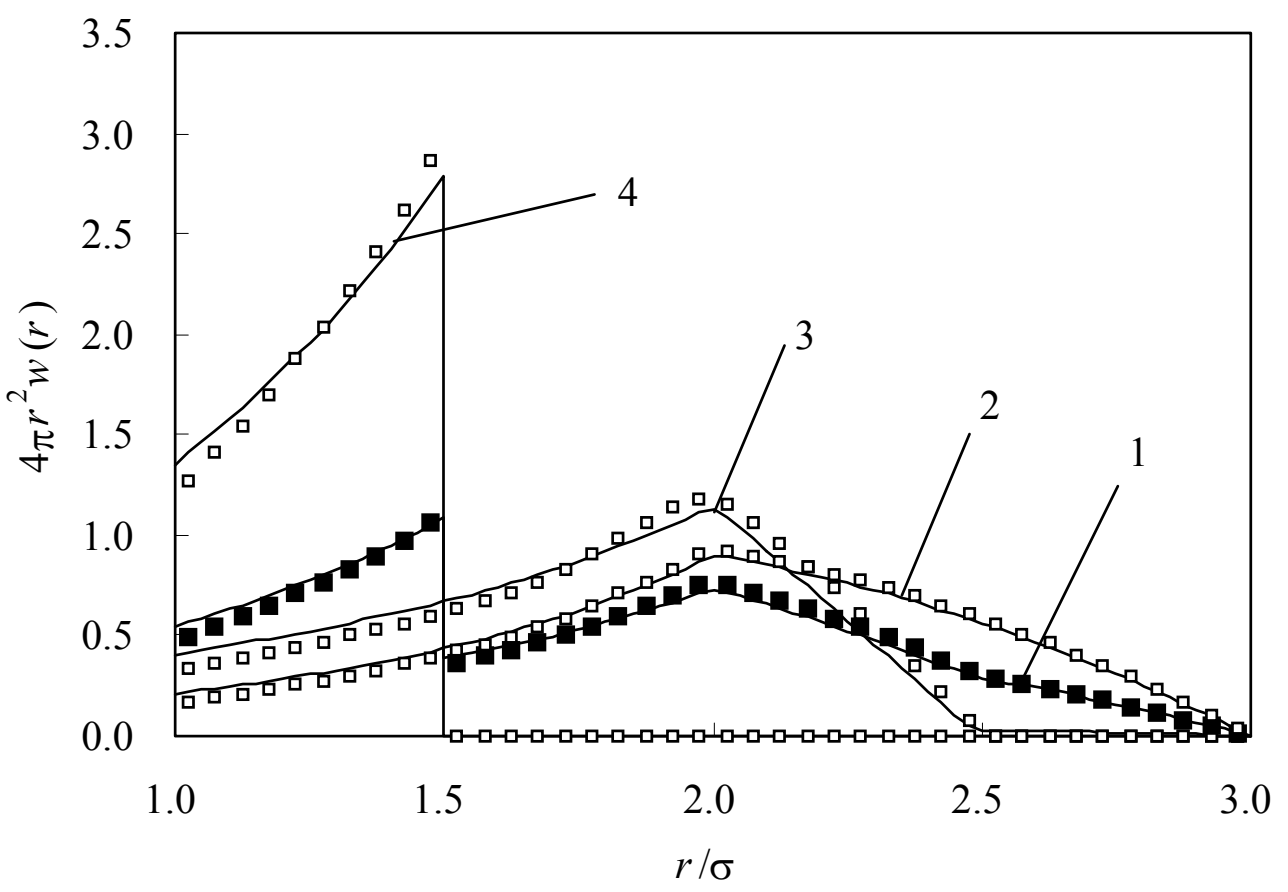


Fig. 6a J. Cai, J. Chem. Phys.

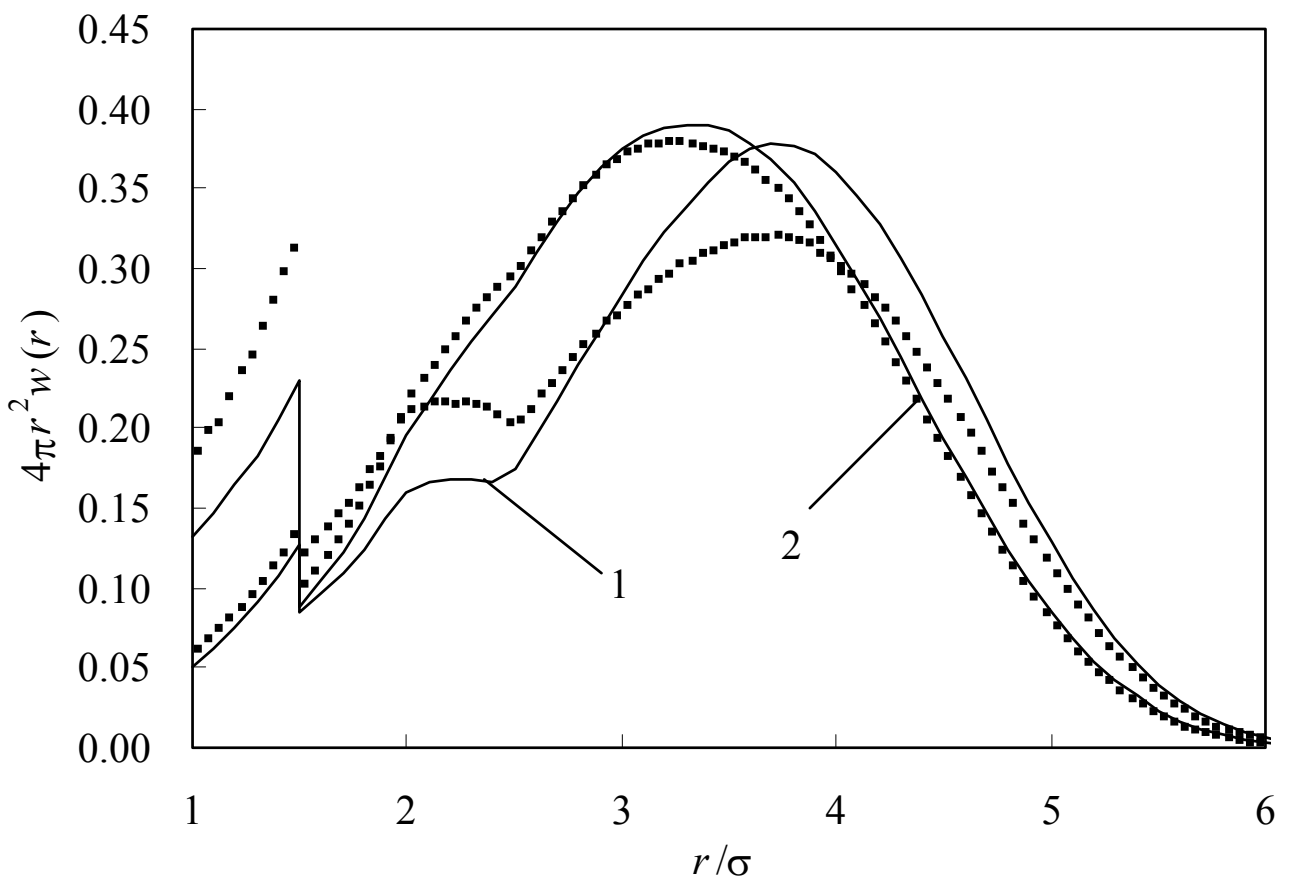


Fig. 6b J. Cai, J. Chem. Phys.

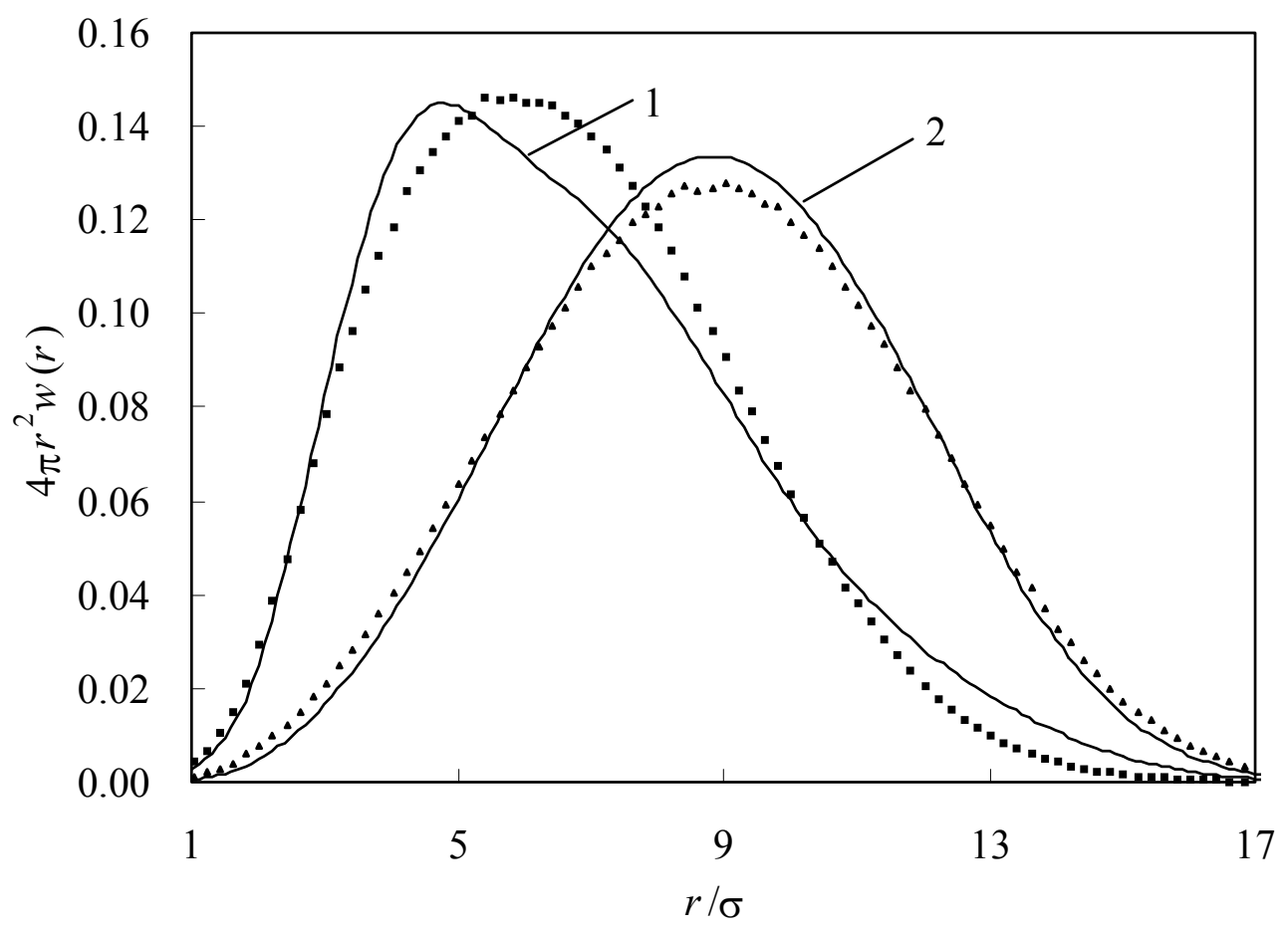


Fig. 7 J. Cai, J. Chem. Phys.

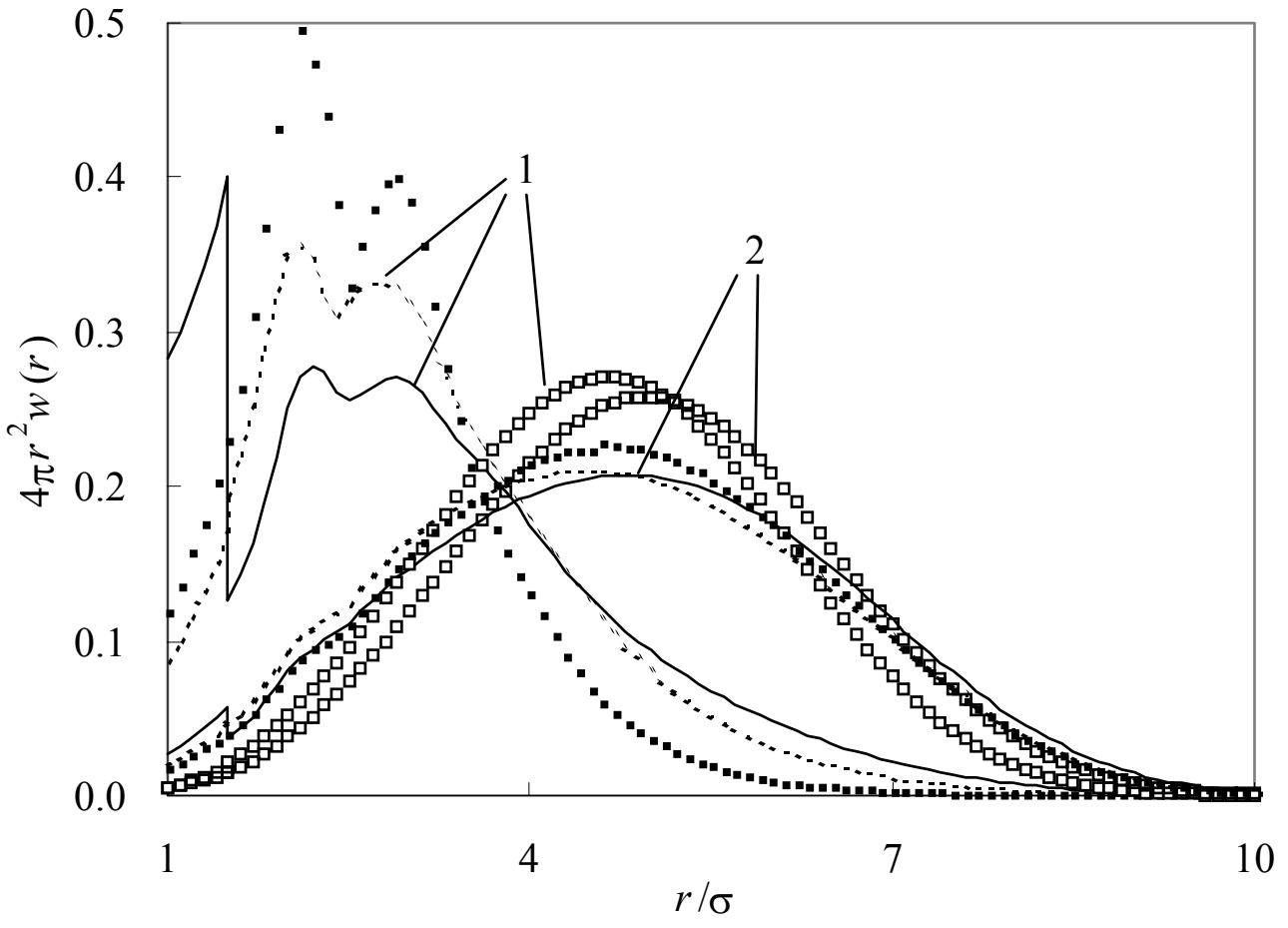


Fig. 8 J. Cai, J. Chem. Phys.

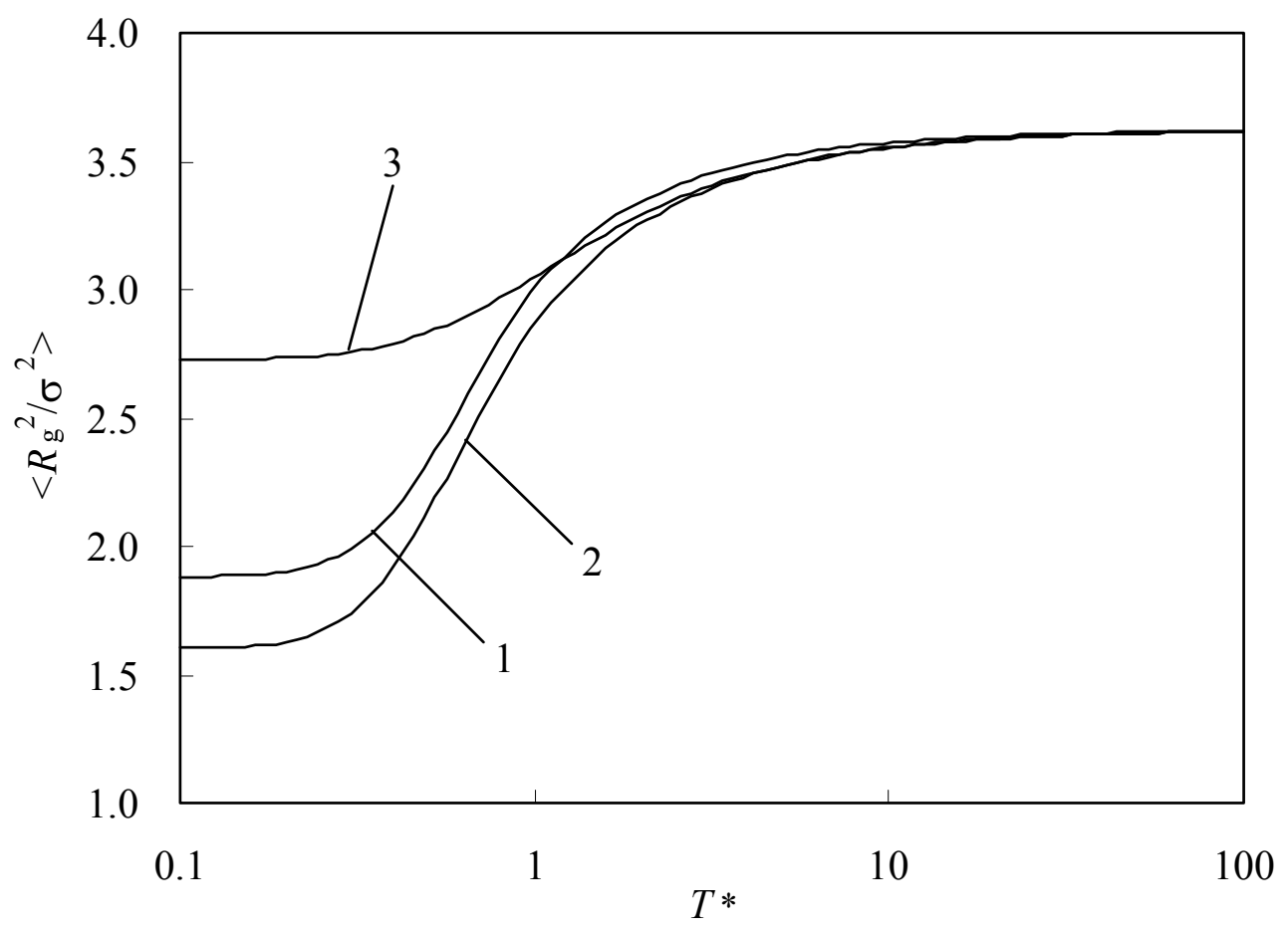

